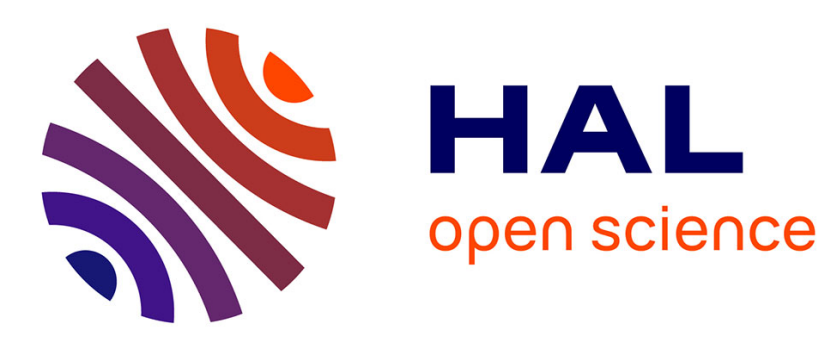

\title{
Analytical formulation of the fractal dimension of filtered stochastic signals
}

Jean-Marc Girault, Denis Kouamé, Abdeldjalil Ouahabi

\section{To cite this version:}

Jean-Marc Girault, Denis Kouamé, Abdeldjalil Ouahabi. Analytical formulation of the fractal dimension of filtered stochastic signals. Signal Processing, 2010, 90 (9), pp.2690 - 2697. 10.1016/j.sigpro.2010.03.019 . hal-01076429

\section{HAL Id: hal-01076429 \\ https://hal.science/hal-01076429}

Submitted on 22 Oct 2014

HAL is a multi-disciplinary open access archive for the deposit and dissemination of scientific research documents, whether they are published or not. The documents may come from teaching and research institutions in France or abroad, or from public or private research centers.
L'archive ouverte pluridisciplinaire HAL, est destinée au dépôt et à la diffusion de documents scientifiques de niveau recherche, publiés ou non, émanant des établissements d'enseignement et de recherche français ou étrangers, des laboratoires publics ou privés. 


\title{
Analytical formulation of the Fractal dimension of filtered stochastic signals
}

\author{
Jean-Marc Girault $^{\mathrm{a}}$, Denis Kouaméb ${ }^{\mathrm{b}}$, Abdeldjalil Ouahabi ${ }^{\mathrm{c}}$ \\ ${ }^{a}$ Université François Rabelais de Tours, UMRS "Imaging and Brain" U930 and CNRS \\ ERL 3106, Tours, France. \\ ${ }^{b}$ Université PAUL Sabatier de Toulouse 3, IRIT UMR 5505, France \\ ${ }^{c}$ Université François Rabelais de Tours, CERTIHS, Tours, France.
}

\begin{abstract}
The aim of this study was to investigate the effects of a linear filter on the regularity of a given stochastic process in terms of the fractal dimension. This general approach, described in a continuous time domain, is new and is characterized by its simplicity. The framework of this problem is general since it emerges when a fractal process undertakes a transformation, as is the case in denoising or measurement processes.
\end{abstract}

Key words: Length, fractal dimension, stochastic signals, Lorentzian filters.

Email address: jean-marc.girault@univ-tours.fr. (Abdeldjalil Ouahabi) 


\section{INTRODUCTION AND DEFINITION}

\subsection{Introduction}

Fractal processes are processes whose ubiquity spans innumerable fields. In contrast to the well-known family of ARMA processes, these intrinsically scale-invariant processes, also known as $1 / f$ processes, are characterized by long-term correlation structure. Moreover, these fractal signals are continuous and underivable signals presenting deterministic or statistical self affinities [27], [29]. Invariants such as the fractal dimension $D$, the Hurst exponent $H$ or the Hölder exponent $h$ are often evaluated to characterize such irregular signals. Stochastic signals can be continuous and not differentiable, presenting strong irregularities.

Our study was performed in the context of analysis and synthesis of fractal signals where fractal and stochastic signals can be related through fractional differentiation [16] or filtering [26]. Others have studied $1 / f$ noise in electronic devices $[3,4,18]$, roughness and texture of surfaces $[12,24,36,17]$ and biomedical signals and images [34, 22, 31, 20].

Issues dealing with filtering and fractal signals have previously been addressed in three ways: the denoising of fractal signals corrupted by an additive noise $[23,35,8,6,7]$, the filtering of fractal signals by a measurement system $[28,12,31,22,36,17,20]$ and the synthesis of fractal signals from stochastic processes $[33,26,10,19,14]$.

In the synthesis of $1 / f^{\beta}$ noise, the framework which predominated for many years was naturally the spectral domain $[3,18,26,33]$. The relationships between the spectral and irregularity properties of the process appeared later in reports by Berry [1] who demonstrated the link between the slope $\beta$ of the spectrum and the fractal dimension $D=(5-\beta) / 2$ for a $\mathrm{fBm}$. The spectral estimator consisted of evaluating the slope $-\beta$ in a log-log plot (Power/frequency), and then applying the previously introduced Berry equation. Although much used in the past, due to its simple formulation (for a continuous or a discrete framework), this spectral estimator is less often used today. The main reasons for its obsolescence are its poor performance and to the need for setting the spectral bandwidth of interest when the power spectral density presents several slopes.

The approaches based on maximum likelihood [23, 35, 7] and the estimators based on multi-scale analysis $[10,35,5,15]$ were mainly used in denoising of fractal signals (or images). Because the likelihood function was 
a non-linear function according to $D$, it was not possible to obtain an explicit formula and only a numerical computation was possible.

For the approaches based on wavelet analysis, they were mainly focused on estimation of the slope of variance $\beta=2 H+1$ of the wavelet coefficients $d_{j}$ in the log-log plot. This estimator was very interesting since it could easily be extended to the study of multi-fractal processes.

When the fractal signals (mono or multidimensional) are accessible only by measurement, as is the case for example when measuring surface roughness $[12,36,17]$ or image texture $[31,22,20]$, the measurement or acquisition systems act like a linear filter. Quantification of the impact of such linear filtering on the regularity of the processes studied is thus necessary. For these applications, the most commonly used fractal dimension estimators have been based on the box counting method (for example see [34]), the spectral slope (for example see [11]) and length measurement [2].

There is not, to our knowledge, a simple framework which directly shows the link between the filter characteristics and the regularity property of such filtered signals through explicit relationships. We therefore investigated the impact of linear filtering on irregularity for a given stochastic process in terms of the fractal dimension.

The novelty of our approach is based on an explicit formulation, in continuous time, linking the spectral properties of a given filter and the fractal properties of a given process. Our approach shows the relationship between the fractal dimensions at the input and the output of a filter. Due to its simple formulation and the good performance of its estimator, we focused on the fractal dimension formulation through the length measurement. The only a priori knowledge required to use our approach is the probability density of the input process and the correlation coefficient of the filter. Our approach is original since the issue has never been previously addressed in denoising, synthesis or measuring processes of fractal signals. Our study is complementary to other studies based mostly on a discrete formulation. The general framework and the simplicity of our formulation are its main assets.

Before explaining the details of our approach, we summarize the definitions used related to the length measurement of fractal stochastic processes.

\subsection{Definition}

The Euclidian length of a deterministic signal $x(t)$ for a limited duration $T$ can be defined as follows: 


$$
L_{0}=T \bar{\lambda},
$$

where

$$
\bar{\lambda}=\frac{1}{T} \int_{-T / 2}^{T / 2} \lambda(t) d t,
$$

is the arithmetic mean of the elementary length and where

$$
\lambda(t)=\sqrt{1+\left(\frac{d x(t)}{d t}\right)^{2}} .
$$

On the hypothesis that $x(t)$ is homogeneous to Volt, then $\bar{\lambda}$ is homogeneous to Volt per second. The elementary length $\lambda$ can be rewritten according to the Pythagorean theorem as:

$$
\lambda(t)=\lim _{\tau \rightarrow 0} \frac{1}{\tau} \sqrt{\tau^{2}+\Delta(t, \tau)^{2}},
$$

where the increment $\Delta(t, \tau)$ is written in a symmetric form as:

$$
\Delta(t, \tau)=x(t+\tau / 2)-x(t-\tau / 2) .
$$

Finally, the signal length is written:

$$
L_{x}=\lim _{\tau \rightarrow 0} \int_{-T / 2}^{T / 2} \frac{1}{\tau} \sqrt{\tau^{2}+\Delta(t, \tau)^{2}} d t .
$$

Note that we had already used such a definition for deterministic signals (see [13]). Unlike deterministic signals, which are almost always derivable and have a finite length, stochastic signals do not follow the laws of Euclidian geometry. Stochastic signals cannot be described by continuous mathematical curves and their irregularities are still present even when examined with higher and higher resolutions. This leads to the paradoxical conclusion that the lengths of such signals are not finite. To overcome the fact that (as fractal signals) stochastic signals do not have a finite length, Richardson [32] assumed that the length $L_{x}$ of a fractal signal depends on the resolution at which it is measured, i.e. the gauge $\lambda$ of the measurement. To overcome this indetermination for $\lambda \rightarrow 0$, Richardson proposed measuring the curve length 
for each value $\lambda, \forall \lambda>0$. Mandelbrot demonstrated that measuring $L_{x}(\lambda)$ as a function of $\lambda$ was one way to determine the fractal dimension:

$$
L_{x}(\lambda)=K_{x} \lambda^{1-D_{x}},
$$

where $D_{x}$ is the fractal dimension and $K_{x}$ is a constant.

Length definition becomes a function of $\lambda$ and is written like equation (1):

$$
L_{x}(\lambda)=T \overline{\lambda(\tau)}
$$

where

$$
\overline{\lambda(\tau)}=\frac{1}{T} \int_{-T / 2}^{T / 2} \lambda(t, \tau) d t
$$

and where the elementary length is $\lambda(t, \tau)=\frac{1}{\tau} \sqrt{\tau^{2}+\Delta(t, \tau)^{2}}$. The corresponding length estimator of equation (8) illustrated in Fig.2 is:

$$
\widehat{L}_{x}\left(k T_{s}\right)=\frac{1}{k T_{s}} \sum_{n=1}^{N-k} \sqrt{\left(k T_{s}\right)^{2}+\Delta\left(n, k T_{s}\right)^{2}},
$$

where $T_{s}$ is the sampling period and $N$ is the number of elementary lengths between $[-T / 2, T / 2]$.

The definition of equation (8) extends easily to stationary and ergodic stochastic signals by replacing the arithmetic mean by the mathematical expectation $E[$.$] . The definition then becomes:$

$$
L_{x}(\lambda)=T E[\lambda]
$$

where $\lambda=\frac{1}{\tau} \sqrt{\tau^{2}+\Delta^{2}}$. Finally, the definition becomes:

$$
L_{x}(\tau)=\frac{T}{\tau} E\left[\sqrt{\tau^{2}+\Delta^{2}}\right] .
$$

The calculation of the mathematical expectation of equation (12) does not lead to an analytical formulation, and only a numerical resolution is possible. In order to present an analytical formulation, we propose using another definition based on that proposed by Burlaga and Klein [2]:

$$
L_{x}(\tau)=\frac{T}{\tau} E[|\Delta|] .
$$


This definition supposes that $\tau<<\Delta$, which is often true, particularly for a high sampling frequency. However, if this is not the case, an amplitude gain can be applied. A discussion of the sense of such a definition is reported in [21].

The length estimator that we finally used which corresponded to a discrete version of equation (13) was:

$$
L_{x}(k)=\sum_{n=1}^{N-k}|\Delta(n, k)| .
$$

The performance of such estimator in its average version, known as the Burlaga and Klein [2] estimator $L_{B K}(k)$, was excellent and outperformed the spectral estimators and the maximum likelihood estimators (see [11]). However, the performance was similar to that obtained with estimators based on wavelet analysis (see [11]). Note that Flandrin [10] demonstrated the link between length measurement $L_{B K}(k)$ and variance of the wavelet coefficient $d_{j}(k): E\left[L_{B K}\left(2^{j-1}\right)\right]=2^{3-5 j / 2} \sqrt{\frac{2}{\pi}} \sqrt{\operatorname{var}\left(d_{j}(k)\right)}$. Though this estimator, which is based on wavelet analysis, gave an excellent performance, it did not lead to a simple formulation in a continuous framework. The strong point of this estimator based on wavelet analysis was its adaptability to study multi-fractal processes.

The paper is organized as follows. Section 2 presents the approach in detail, setting out the notion of length ratio of colored processes, then stating the link between the length ratio and the fractal dimension of colored processes. Section 3 presents several examples of filters and the simulation results are discussed. Finally we propose a conclusion and some future applications.

\section{LENGTH RATIO AND FRACTAL DIMENSION OF FILTERED STOCHASTIC SIGNALS}

The method that we propose is new and was investigated in a general stochastic framework with an explicit formulation in continuous time. This approach requires some a priori information such as the probability density of the input process and the correlation function of the filter.

In a linear system where the impulse response is $h(t)$, the input signal $x(t)$ and the output signal $y(t)$, and the length $L_{x}(\tau)$ of the input signal, can be evaluated by: 


$$
L_{x}(\tau)=\frac{T}{\tau} E\left[\left|x_{1}-x_{2}\right|\right],
$$

where $x_{1}=x\left(t_{1}+\tau / 2\right), x_{2}=x\left(t_{2}-\tau / 2\right) \forall t_{1}, t_{2}$ and where

$$
E\left[\left|x_{1}-x_{2}\right|\right]=\iint\left|x_{1}-x_{2}\right| p_{x_{1}, x_{2}}\left(x_{1}, x_{2}\right) d x_{1} d x_{2} .
$$

$p_{x_{1}, x_{2}}\left(x_{1}, x_{2}\right)$ is the joint probability density.

\subsection{Filtered Gaussian noise}

In the special case where $x_{1}$ and $x_{2}$ are two random Gaussian variables, the joint probability density $p_{x_{1}, x_{2}}\left(x_{1}, x_{2}\right)$ is written:

$$
p_{x_{1}, x_{2}}\left(x_{1}, x_{2}\right)=\frac{\left(1-\rho_{x}^{2}\right)^{-1 / 2}}{2 \pi \sigma_{1} \sigma_{2}} e^{\frac{-1}{2\left(1-\rho_{x}^{2}\right)}\left(\frac{x_{1}^{2}}{\sigma_{1}^{2}}+\frac{x_{2}^{2}}{\sigma_{2}^{2}}-\frac{2 \rho_{x} x_{1} x_{2}}{\sigma_{1} \sigma_{2}}\right)},
$$

where $\sigma_{1}^{2}$ and $\sigma_{2}^{2}$ are the variances of the two random variables $x_{1}$ and $x_{2}$, respectively. $\rho_{x}$ is the correlation coefficient defined by $\rho_{x}=E\left[x_{1} x_{2}\right] / E\left[x_{1}^{2}\right] E\left[x_{2}^{2}\right]$.

The probability density of the increment $\Delta=x_{2}-x_{1}$ is written:

$$
p_{\Delta}(\Delta)=\int_{-\infty}^{+\infty} p_{x_{1} x_{2}}\left(x_{1}, x_{1}+\Delta\right) d x_{1}
$$

After calculation, the probability density of the increment is reduced to a normal law $N\left(0, \sigma^{\prime 2}\right)$ :

$$
p_{\Delta}(\Delta)=\frac{1}{\sqrt{2 \pi} \sigma^{\prime}} e^{\frac{-\Delta^{2}}{2 \sigma^{\prime 2}}}
$$

with $\sigma^{\prime}=\sqrt{\sigma_{1}^{2}+\sigma_{2}^{2}-2 \rho_{x} \sigma_{1} \sigma_{2}} . \quad x_{1}$ and $x_{2}$ belong to the same signal $x(t)$, then $\sigma_{1}=\sigma_{2}=\sigma_{x}$ and the standard deviation $\sigma^{\prime}$ is simplified, $\sigma^{\prime}=\sigma_{x} \sqrt{2\left(1-\rho_{x}\right)}$.

The mathematical expectation of the increment becomes:

$$
E[|\Delta|]=2 \int_{0}^{+\infty} \Delta p_{\Delta}(\Delta) d \Delta=\sqrt{\frac{2}{\pi}} \sigma^{\prime},
$$

and finally:

$$
L_{x}(\tau)=\frac{2}{\sqrt{\pi}} \frac{T \sigma_{x}}{\tau} \sqrt{1-\rho_{x}(\tau)} .
$$


Similarly the length of the output signal $y(t)$ is written:

$$
L_{y}(\tau)=\frac{2}{\sqrt{\pi}} \frac{T \sigma_{y}}{\tau} \sqrt{1-\rho_{y}(\tau)} .
$$

Note that for a white noise $\left(\rho_{x}=0\right)$, the length of the input signal $x(t)$ is written:

$$
L_{x}(\tau)=\frac{T \sigma_{x}}{\tau} \gamma
$$

where the factor $\gamma$ is equal to $2 / \sqrt{\pi}$ for a Gaussian noise and the factor $\gamma$ is equal to $\frac{2}{\sqrt{3}}$ for a noise uniformly distributed between $[-a / 2, a / 2]$ with a standard deviation $\sigma_{x}=a / \sqrt{12}$. The factor $\gamma$ (and hence the length) seems to reveal the statistic nature of the white noise.

In order to establish a link between the impulse response or the correlation function of the filter $\rho_{y}(\tau)$ and the fractal dimension $D_{y}$ of the filtered signal $y(t)$, it should be remembered that in the case of fractal signals, the length $L_{y}$ of the output signal is related to the fractal dimension $D_{y}$ as follows (see also equation (7) for the input):

$$
L_{y}(\tau)=K_{y} \tau^{1-D_{y}},
$$

where $K_{y}$ is a constant. Consequently, the fractal dimension $D_{y}$ of the signal $y(t)$ can be defined as follows:

$$
D_{y}=1-\lim _{\tau \rightarrow 0} \frac{\log \left(L_{y}(\tau)\right)}{\log (\tau)} .
$$

Similarly:

$$
D_{x}=1-\lim _{\tau \rightarrow 0} \frac{\log \left(L_{x}(\tau)\right)}{\log (\tau)} .
$$

By using equations (20), (25) and (21),(24) the equations become:

$$
\begin{aligned}
& D_{y}=2-\lim _{\tau \rightarrow 0} \frac{1}{2} \frac{\log \left(1-\rho_{y}(\tau)\right)}{\log (\tau)}, \\
& D_{x}=2-\lim _{\tau \rightarrow 0} \frac{1}{2} \frac{\log \left(1-\rho_{x}(\tau)\right)}{\log (\tau)} .
\end{aligned}
$$


For unidimensional signal, the fractal dimension of signals $x(t)$ and $y(t)$ was the topological dimension of the space minus a term related to the inner correlation of $x(t)$ and $y(t)$.

In order to simply qualify the influence of linear filters, we propose evaluating a new parameter, i.e. the length ratio $\Omega$ between the input and the output of the linear system:

$$
\Omega(\tau)=\frac{L_{y}(\tau)}{L_{x}(\tau)} .
$$

By using equations (20) and (21), equation (28) becomes:

$$
\Omega(\tau)=\frac{\sigma_{y}}{\sigma_{x}} \sqrt{\frac{1-\rho_{y}(\tau)}{1-\rho_{x}(\tau)}} .
$$

Furthermore, the following function $\Lambda(\tau)$ is defined:

$$
\Lambda(\tau)=\lim _{\tau \rightarrow 0} \frac{\log (\Omega(\tau))}{\log (\tau)} .
$$

$\Lambda(\tau)$ is an important parameter since it quantifies the filtering impact in terms of reduction irregularity. By using equations (7), (23) and (28), it is easy to show that:

$$
\Lambda=D_{x}-D_{y}
$$

In this case $D_{y}$ is defined by:

$$
D_{y}=D_{x}-\lim _{\tau \rightarrow 0} \frac{\log (\Omega(\tau))}{\log (\tau)} .
$$

When the input signal is a Gaussian noise and when the system is linear, by using equations (29) and (32), this becomes:

$$
D_{y}=D_{x}-\lim _{\tau \rightarrow 0}\left(\frac{1}{2} \frac{\log \left(1-\rho_{y}(\tau)\right)}{\log (\tau)}\right)+\lim _{\tau \rightarrow 0}\left(\frac{1}{2} \frac{\log \left(1-\rho_{x}(\tau)\right)}{\log (\tau)}\right) .
$$

The fractal dimension of the filtered signal is then equal to the fractal dimension of the signal before filtering minus a term related to the filter plus a term explaining the inner correlation of the input signal $x(t)$.

Before testing different types of filter, we present the filtered fractional Brownian motion. 


\subsection{Filtered fractional Brownian motion}

Let's consider the fractional Brownian motion ( $\mathrm{fBm}$ ) [25] as being in the input of the filter. This self similar process which generalizes the Brownian motion or the Wiener process is composed of stationary and independent increments. These increments follow a Gaussian law ${ }^{1}: x_{1}-x_{2} \approx N\left(0,2 \tau^{2 H_{x}}\right)$ where $H_{x}$ is the Hurst exponent. Note that an average power spectrum density of fBm can be defined in the frequency domain [9] and it turns out that it is proportional to $1 / f^{\alpha}$ with $\alpha=2 H_{x}+1$. By using equation (14) to equation (20), we show that:

$$
L_{x}(\tau)=\frac{2}{\sqrt{\pi}} T \tau^{H_{x}-1} .
$$

Note that we recognize the factor $2 / \sqrt{\pi}$ which underlines the Gaussian nature of the process. By using equations (7-25), it becomes:

$$
D_{x}=2-H_{x},
$$

where

$$
H_{x}=1+\lim _{\tau \rightarrow 0} \frac{\log \left(L_{x}(\tau)\right)}{\log (\tau)} .
$$

Note that $H_{y}$ has the same definition as $H_{x}$. As for equation (33), it becomes:

$$
H_{y}=H_{x}-\lim _{\tau \rightarrow 0}\left(\frac{1}{2} \frac{\log \left(1-\rho_{y}(\tau)\right)}{\log (\tau)}\right) .
$$

Similarly, the Hurst exponent $H_{y}$ of the filter output is the Hurst exponent $H_{x}$ before filtering minus a term related to the inner filter properties.

\subsection{Impact of the correlation coefficient}

From definitions (33) and (37), it becomes obvious that the asymptotic behavior of the correlation coefficient when $\tau \rightarrow 0$ is a key point in the estimation of fractal dimension. Indeed, by exciting filters by a white Gaussian

\footnotetext{
${ }^{1}$ Note that the increments follow a Gaussian law written as follows: $x_{1}-x_{2} \approx$ $N\left(0, \tau^{2 H_{x}}\right)$. However, in order to make a direct link between equations in this subsection and equations in subsection 2.1, we voluntary modified this formula by doubling the variance and this modification does not modify the definition of $\mathrm{fBm}$.
} 
noise, all correlation coefficients $\rho_{y}(\tau)$ whose Taylor expansion satisfies the equation:

$$
\lim _{\tau \rightarrow 0} \rho_{y}(\tau) \approx 1-k \tau^{n},
$$

where $k$ is a known constant and where $n \in \mathbb{R}$ and $0 \leq n \leq 2$, involve a fractal dimension and a Hurst exponent described as follows:

$$
\left\{\begin{array}{l}
D_{y}=D_{x}-\frac{n}{2} \\
H_{y}=H_{x}+\frac{n}{2}
\end{array}\right.
$$

where $D_{x}=2$ and $H_{x}=0$ for $\rho_{x}=0$. Note that for $2 \geq D_{y} \geq 1, n$ satisfies $0 \leq n \leq 2$. Moreover, by using definition (33) and equation (38) for $\rho_{x}=0$, it becomes:

$$
D_{y}=2-\lim _{\tau \rightarrow 0} \frac{1}{2} \frac{\log \left(k \tau^{n}\right)}{\log (\tau)}=2-\frac{n}{2},
$$

and $n=4-2 D_{y}$. Without making out an exhaustive list, we propose the following correlation coefficients (or a combination) satisfying equation (38) for $\tau>0$ :

- $\rho_{y}(\tau)=\alpha+(1-\alpha) \cos \left(k \tau^{n / 2}\right)$. For $\alpha=0.5$ and for $n=2$ we recognize the Hanning function;

- $\rho_{y}(\tau)=\left(1-k \tau^{n}\right)$. For $n=1$ we recognize the triangle function;

- $\rho_{y}(\tau)=e^{-k \tau^{n}}$. For $n=2$ we recognize the Gaussian function and for $n=1$ we recognize the Lorentzian function.

\section{EXAMPLES}

\subsection{Lorentzian filters}

The impulse response $h(t)$ of a Lorentzian filter is defined by:

$$
h(t)=\omega_{c} e^{-\omega_{c} t} \cos \left(\omega_{0} t\right) u(t),
$$

where $u(t)$ is the Heaviside function, $\omega_{c}$ the cut-off pulsation and $\omega_{0}$ the central pulsation of the filter. This linear system of impulse response $h(t)$ is excited by a Gaussian white noise. For linear filters, the crosscorrelation function of the output signal is written: 


$$
R_{y}(\tau)=R_{h}(\tau) * R_{x}(\tau),
$$

where $*$ is the convolution operator. Note that the process coming from this type of filter is also known as being the "first order Markov process". For the Lorentzian filter the correlation function is $R_{h}(\tau)=\frac{\omega_{c}}{4} e^{-\omega_{c}|\tau|} \cos \left(\omega_{0} \tau\right)$, the correlation function of the input signal is $R_{x}(\tau)=\sigma_{x}^{2} \delta(\tau)$, and the correlation function of the output signal reduces to $R_{y}(\tau)=\sigma_{y}^{2} e^{-\omega_{c}|\tau|} \cos \left(\omega_{0} \tau\right)$, where $\sigma_{y}=\sigma_{x} \sqrt{\omega_{c}} / 2$. The correlation coefficient defined by $\rho_{y}(\tau)=R_{y}(\tau) / R_{y}(0)$, is reported in Table I for $\tau>0$.

As already mentioned, the length of the white Gaussian noise is $\forall \tau>0$ :

$$
L_{x}(\tau)=\frac{2 T \sigma_{x}}{\sqrt{\pi} \tau}
$$

since the correlation coefficient is $\rho_{x}=0, \forall \tau>0$. The length of the output signal is $\forall \tau>0$ :

$$
L_{y}(\tau)=\frac{2 T \sigma_{y}}{\sqrt{\pi} \tau} \sqrt{1-e^{-\omega_{c} \tau} \cos \left(\omega_{0} \tau\right)} .
$$

The length ratio $\forall \tau>0$ is written as follows:

$$
\Omega(\tau)=G \sqrt{1-e^{-\omega_{c} \tau} \cos \left(\omega_{0} \tau\right)},
$$

where the gain $G$ is written $G=\sigma_{y} / \sigma_{x}$.

In order to test different kinds of Lorentzian filter, four filters were considered: a wide band filter, a narrow band filter, a base band filter and an all pass filter. The correlation coefficients and length ratios for these different kinds of filter are summarized in Table I. We verified that for these different kinds of filter, the parameter $\Omega / G$ was related to the intrinsic filter parameters, $\omega_{c}$ and $\omega_{0}$.

When the input is a white Gaussian noise, the fractal dimension $D_{y}$ of the output is:

$$
D_{y}=2-\lim _{\tau \rightarrow 0}\left(\frac{1}{2} \frac{\log \left(1-e^{-\omega_{c} \tau} \cos \left(\omega_{0} \tau\right)\right)}{\log (\tau)}\right) .
$$

- For a base band filter $\omega_{0} \rightarrow 0$ and for $\tau \rightarrow 0, \omega_{c} \tau<<1$ :

$$
D_{y}=2-\frac{1}{2}=\frac{3}{2}
$$


with

$$
\lim _{\tau \rightarrow 0}\left(1-e^{-\omega_{c} \tau}\right) \approx \omega_{c} \tau
$$

- For a narrow band filter $\omega_{c} \rightarrow 0$ and for $\tau \rightarrow 0, \omega_{0} \tau<<1$ :

$$
D_{y}=2-1=1
$$

with

$$
\lim _{\tau \rightarrow 0}\left(1-\cos \left(\omega_{0} \tau\right)\right) \approx \omega_{0}^{2} \tau^{2} / 2 .
$$

The output signal is a sinusoid, i.e. a continuous signal whose fractal dimension is the same as a line. Note that for a Gaussian correlation coefficient $\rho_{y}(\tau)=e^{-\omega_{c} \tau^{2}}$, the fractal dimension is also $D_{y}=1$ since $\lim _{\tau \rightarrow 0}\left(1-e^{-\omega_{c} \tau^{2}}\right) \approx \omega_{c} \tau^{2}$.

- For an all pass filter:

$$
D_{y}=2-\lim _{\tau \rightarrow 0}\left(\frac{1}{2} \frac{\log (1)}{\log (\tau)}\right)=2-0=2 .
$$

The output signal is a white noise, i.e. an irregular signal which fills all the space.

When the input is a fractional Brownian motion, the Hurst exponent of the output signal is:

- for a base band filter $\omega_{0} \rightarrow 0$ and for $\tau \rightarrow 0, \omega_{c} \tau<<1$ :

$$
H_{y}=H_{x}+\lim _{\tau \rightarrow 0}\left(\frac{1}{2} \frac{\log \left(\omega_{c} \tau\right)}{\log (\tau)}\right) .
$$

By imposing $H_{x}=0.3$ (high irregularity), the Hurst exponent of the output is: $H_{y}=0.3+0.5=0.8$. This confirms the fact that the greater the filtering the greater the regularity.;

- for a narrow band filter $\omega_{c} \rightarrow 0$ and for $\tau \rightarrow 0, \omega_{0} \tau<<1$ :

$$
H_{y}=H_{x}+\lim _{\tau \rightarrow 0}\left(\frac{1}{2} \frac{\log \left(\omega_{0}^{2} \tau^{2} / 2\right)}{\log (\tau)}\right) .
$$

By imposing $H_{x}=0.3$ (high irregularity), the Hurst exponent of the output is: $H_{y}=0.3+1=1.3$. As $0 \leq H_{y} \leq 1$, this means that $H_{y}=1$. 


\subsection{Simulations}

As an illustration, Figs. 3 and 4 show the length ratios for the four types of Lorentzian filter. Time series of 10000 samples are simulated with a sampling frequency of $10000 \mathrm{~Hz}$ for different values of the cut-off frequency $f_{c}=$ $\{1,10,100,1000,5000\} H z$ and for different values of the central frequency of the filter $f_{0}=\{1,10\} \mathrm{Hz}$. The minimum and maximum values of the gauge are $\tau_{\min }=10^{-4} \mathrm{~s}$ and $\tau_{\max }=0.5 \mathrm{~s}$. The sampling frequency is thus an important setting parameter since it fixes the minimum resolution.

Fig. 3 shows that for a cut-off frequency $f_{c}=5000 \mathrm{~Hz}$ and a central frequency $f_{0}=1 \mathrm{~Hz}$ (similar to an all pass filter), the length ratio $\Omega$ of such a signal has a slope equal to zero, involving a fractal dimension $D_{y}=2$. The output signal is a white Gaussian noise which entirely fills the space. Note that for a white noise, we verified that $\Omega\left(\tau_{\text {min }}\right) / G \approx 1$. When the cut-off frequency was reduced to $f_{c}=1 \mathrm{~Hz}$ and $f_{0}=1 \mathrm{~Hz}$ (low pass filter), the slope for the length ratio was $1 / 2$ for a gauge lower than the cut-off gauge $\tau<\tau_{c}=1 / \omega_{c}$, involving a fractal dimension $D_{y}=3 / 2$. The output signal was a standard Brownian motion characterized by its Hurst exponent $H=1 / 2$. Beyond the cut-off gauge, the slope was equal to zero.

Fig. 4 shows that for a central frequency $f_{0}=100 \mathrm{~Hz}$ and for a cut-off frequency $f_{c}=10 \mathrm{~Hz}$, oscillations where the position of its first minimum corresponds to $\tau=1 / f_{0}$ appear in $\Omega$. For a gauge less than the cut-off gauge $\tau_{c}=1 / \omega_{c}$, the fractal dimension is between 1.5 and 1 for a narrow band filter and between 2 and 1.5 for a wide band filter. When the cut-off frequency tends towards zero, the fractal dimension tends to 1, thus involving the presence of a sinusoidal signal. When the cut-off frequency tends towards infinity (whatever the central frequency) then the fractal dimension tends to 2 , involving the presence of a white noise.

By using an impulse response of the form $h(t)=e^{-\omega_{c} \tau^{n}}$ with $0 \leq n \leq 1$, time series of 5000 samples are simulated with a sampling frequency of $10000 \mathrm{~Hz}$ and a cut-off frequency $f_{c}=1 \mathrm{~Hz}$. The input signal is a white Gaussian noise $N(0,1)$. The minimum and maximum values of the gauge are $\tau_{\min }=10^{-4} \mathrm{~s}$ and $\tau_{\max }=0.5 \mathrm{~s}$. Simulation results are depicted in figure 5. Figure 5a shows synthesized fractal signals: the higher the order $n$, the greater the regularity of the time series. Figure $5 \mathrm{~b}$ shows the length ratio $\Omega / G$ with respect to the gauge $\tau$ : the higher the order $n$, the higher the slope and the lower the fractal dimension. For $n=0$, the corresponding filter is an all pass filter, the output signal is a white Gaussian noise and the corresponding fractal dimension is $D_{y}=2$. For $n=1$, the corresponding filter is a base 
band filter, the output signal is a Brownian motion and the corresponding fractal dimension is $D_{y}=1.5\left(H_{y}=0.5\right)$. For $0 \leq n \leq 1$ the corresponding fractal dimensions of the output filter are between $2 \geq D_{y} \geq 1.5$.

\section{DISCUSSION \& CONCLUSION}

We propose here a simple explicit formulation of the filtering effect on the regularity of a given stochastic process in a general framework and in a continuous time context. The simulations studied validated the theoretical calculus but also permitted extraction of the filter characteristics such as cut-off frequency and central frequency.

Though limited to mono-fractal processes, this approach is interesting since it requires only commonly used a priori knowledge such as the probability law of the process and the spectral characteristic of the filter. The need for a priori information such as the probability density and the correlation coefficient of the filter constitute both an advantage and a drawback. It is an advantage since they provide a simple formulation, but it is also a disadvantage since the study presented here was limited to this information. Another drawback is that our approach is currently limited to mono-fractal processes. The next step will be to extend our framework to the study of multi-fractal processes.

\section{References}

[1] M.V. Berry, Diffractals, J. Phys. A Math Gen, Vol 12, pp 781, 1979.

[2] L. Burlaga and L. Klein, Fractal structure of the interplanetary magnetic field, Journal of Geophysical Research, Vol 91, pp 347-350, 1986.

[3] J.A. Barnes and D.W. Allan, A statistical model of flicker noise, Proc. IEEE, Vol 54, pp 176-185, 1966.

[4] J.A. Barnes and S. Jarvis, Efficient numerical and analog modeling of flicker noise processes, National Bureau od standard Technical Note 604, 1971.

[5] B.S. Chen and G. W. Lin, Multiscale Wiener filter for the restoration of fractal signals: Wavelet filter bank approach, IEEE Trans. Signal Processing, Vol 42, pp 2972-2982, 1994. 
[6] B.S. Chen and W.S. Hou, Deconvolution filter desing for fractal signal transmission systems: a multiscale Kalman filter bank approach, IEEE Trans. Signal Processing, Vol 45, pp 1359-1364, 1997.

[7] B.S. Chen and B.K. Lee and S.C. Peng, Maximum Likelihood Parameter Estimation of F-ARIMA Processes Using the Genetic algorithm in the Frequency Domain, IEEE Trans. Signal Processing, Vol 50, pp 2208$2220,2002$.

[8] M. Deriche and A.H. Tewfik, Signal Modeling with filtered discrete fractional noise processes, IEEE Trans. signal Processing, Vol 41, pp 2839 2849, 1993.

[9] P. Flandrin, On the spectrum of Fractional Brownian Motions, IEEE Trans. Inform. Theory, Vol 35, pp 197-199, 1989.

[10] P. Flandrin, Wavelet analysis and synthesis of fractional Brownian motion, IEEE Trans. Inform. Theory, Vol 38, pp 910-917, 1992.

[11] N. Gache and P. Flandrin and D. Garreau, Fractal Dimension Estimators for Fractional Brownian Motions, Proc. ICASSP, pp 3557-3560, 1991.

[12] J.J Gagnepain and C. Roques-Carmes, Fractal approach to twodimensional and three-dimensional surface roughness, International conference on metrology and properties of engineering surfaces, Vol 109, pp 19-126, 1986.

[13] J.M. Girault and D. Kouamé, Length and frequency of band-limited signals, IEEE signal Processing Letters, Vol 9, pp 371-374, 2002.

[14] M. Guglielmi, 1/f ${ }^{\alpha}$ signal synthesis with precision control, Signal Processing, Vol 86, pp 2548-2553, 2006.

[15] G.A. Hirchoren and C.E. D'Attellis, On the Optimal number of scales in estimation of fractal signals using wavelets and filter banks, Signal Processing, Vol 63, pp 55-63, 1997.

[16] J.R.M. Hosking, Fractional differencing, Biometrika, Vol 68, pp 165-176, 1981. 
[17] J. James, Fracture surface examination of dental ceramics using fractal analysis, Dental Materials, Vol 21, pp 586-589, 2005.

[18] M.S. Keshner, 1/f noise, Proc. IEEE, Vol 70, pp 211-219, 1982.

[19] N.J. Kasdin, Discrete simulation of colored noise and stochastic process and $1 / f$ power law noise generation, Proc. IEEE, Vol 83, pp 802-826, 1995.

[20] W.-L. Lee and K.-S. Hsieh, A robust algorithm for the fractal dimension of images and its applications to the classification of natural images and ultrasonic liver images, Signal Processing, in press, 2009.

[21] E. Le Tavernier, La méthode de Higuchi pour la dimension fractal, Signal Processing, Vol 65, pp 115-128, 1998.

[22] T. Loussot and R. Harbar and G. Jacquet and C.L. Benhamou and E. Lespesailles and A. Julien, An oriented fractal analysis for the characterization of texture: application to bone radiographs, Eusipco Signal Processing, Vol 1, pp 371-374, 1996.

[23] T. Lundahl and W.J. Ohley and S.M. Kay and R. Siffert, Fractional Brownian Motion: A maximum likelihood estimator and its application to image texture, IEEE Trans. Med. Imaging, Vol 5, pp 151-161, 1986.

[24] A. Majumdar and B. Bhushan, Fractal model of elastic-plastic contact between rough surfaces, Journal of Tribology, Vol 113, pp 1-11, 1991.

[25] B.B. Mandelbrot and J.W. Van Ness, Fractional Brownian Motions, Fractional Noises and applications, SIAM Rev., Vol 10, pp 422-437, 1968.

[26] B.B. Mandelbrot, A Fast Fractional Gaussian Noise Generator, Water Resource Research, Vol 7, pp 543-553, 1971.

[27] B. Mandelbrot, The fractal geometry of nature, San Francisco-Freeman, 1982.

[28] B.B. Mandelbrot and D.E. Passoja and A.J. Paulay, Fractal character of fracture surfaces of metals, Nature, Vol 308, pp 721-722, 1984. 
[29] B. Mandelbrot, Self-affine fractals and fractal dimension, Physica Scripta, Vol 32, pp 257-260, 1986.

[30] H.O. Peitgen and D. Saupe, The Science of Fractal Images, SpringerVerlag, New-York, 1988.

[31] P. Qingrui and Y. Zhuangzhi, Mammogram analysis based on wavelets and fractals, Proceedings of IEEE Engineering in Medecine and Biology society, pp 1159-1162, 1996.

[32] L. Richardson, The problem of contiguity: an appendix of statistic of deadly quarrels, General systems yearbook, Vol 61, pp 139-187, 1961.

[33] R. Saletti, A comparison between two methods to generate $1 / f$ noise, Proc. IEEE, Vol 74, pp 1595-1600, 1986.

[34] N. Sarkar and B.B. Chaudhuri, An efficient differential box-counting approach to compute fractal dimension of image, Ieee Trans. Sys. Man Cybernetics, Vol 24, pp 115-120, 1994.

[35] G. W. Wornell and A. V. Oppenheim, Estimation of fractal signals from noisy measurements using Wavelets, IEEE Trans. Signal Processing, Vol 40, pp 611-622, 1992.

[36] J.J. Wu, Characterization of fractal surfaces, Wear, Vol 239, pp 36-47, 2000. 
List of Tables 
Table I

Correlation coefficients and length ratios $\forall \tau>0$.

\begin{tabular}{|c||c|c|c|}
\hline \hline Filters & $h(t)$ & $\rho_{y}(\tau)$ & \multicolumn{2}{|c|}{$\Omega(\tau) / G$} \\
\hline \hline Wide band & $\omega_{c} e^{-\omega_{c} t} \cos \left(\omega_{0} t\right) u(t)$ & $e^{-\omega_{c} \tau} \cos \left(\omega_{0} \tau\right)$ & $\sqrt{1-e^{-\omega_{c} \tau} \cos \left(\omega_{0} \tau\right)}$ \\
\hline Narrow band & $\cos \left(\omega_{0} t\right) u(t)$ & $\cos \left(\omega_{0} \tau\right)$ & \multicolumn{2}{|c|}{$\sin \frac{\omega_{0} \tau}{2} \sqrt{2}$} \\
\hline Base band & $\omega_{c} e^{-\omega_{c} t} u(t)$ & $e^{-\omega_{c} \tau}$ & \multicolumn{2}{|c|}{$\sqrt{1-e^{-\omega_{c} \tau}}$} \\
\hline All pass & $\delta(t)$ & 0 & \multicolumn{1}{|c|}{1} \\
\hline \hline
\end{tabular}

where $\rho_{x}=0 \forall \tau>0$ and where the gain is $G=\sigma_{y} / \sigma_{x}$. 


\section{List of Figures}

1 Low pass filtering increases the regularity of the fractal signal.

a) Fractal signal $(H=0.5)$. b) Filtered fractal signal. . . . . . 22

2 Principle of length measurement of a stochastic signal. . . . . 23

3 Length ratio of colored stochastic signals. First order Lorentzian low pass filter for different cut-off pulsations and for a fixed central pulsation. . . . . . . . . . . . . . . . . . 24

4 Length ratio of colored stochastic signals. First order Lorentzian low pass filter for different cut-off pulsations and for a fixed central pulsation. . . . . . . . . . . . . . . 25

5 Length ratio of colored stochastic signals. a) Synthesized fractal signals with an $n$-order "Lorentzian filter". b) Loglog plot of Length ratio $\Omega / G$ versus gauge $\tau$. . . . . . . . . . . . . 26 
Fractal signal $(\mathrm{H}=0.5)$ and filtered fractal signal
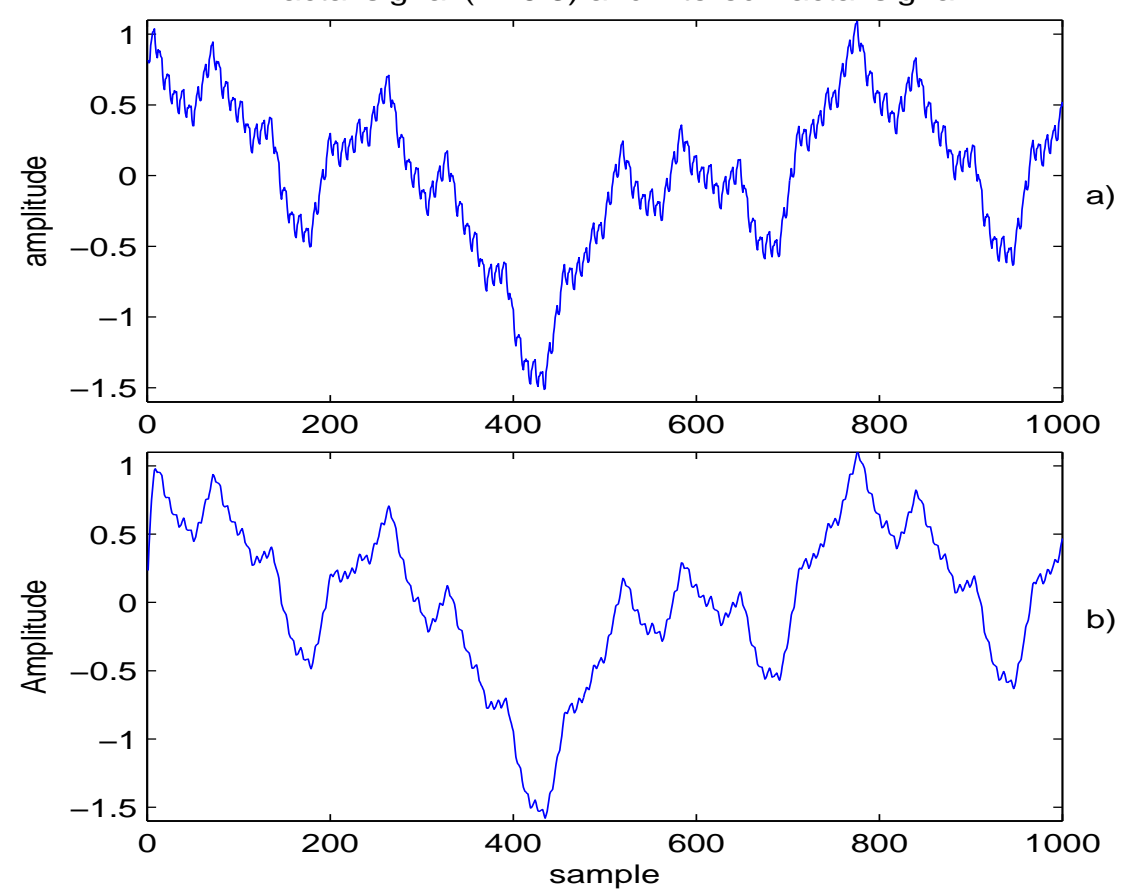

Figure 1: Low pass filtering increases the regularity of the fractal signal. a) Fractal signal $(H=0.5)$. b) Filtered fractal signal. 


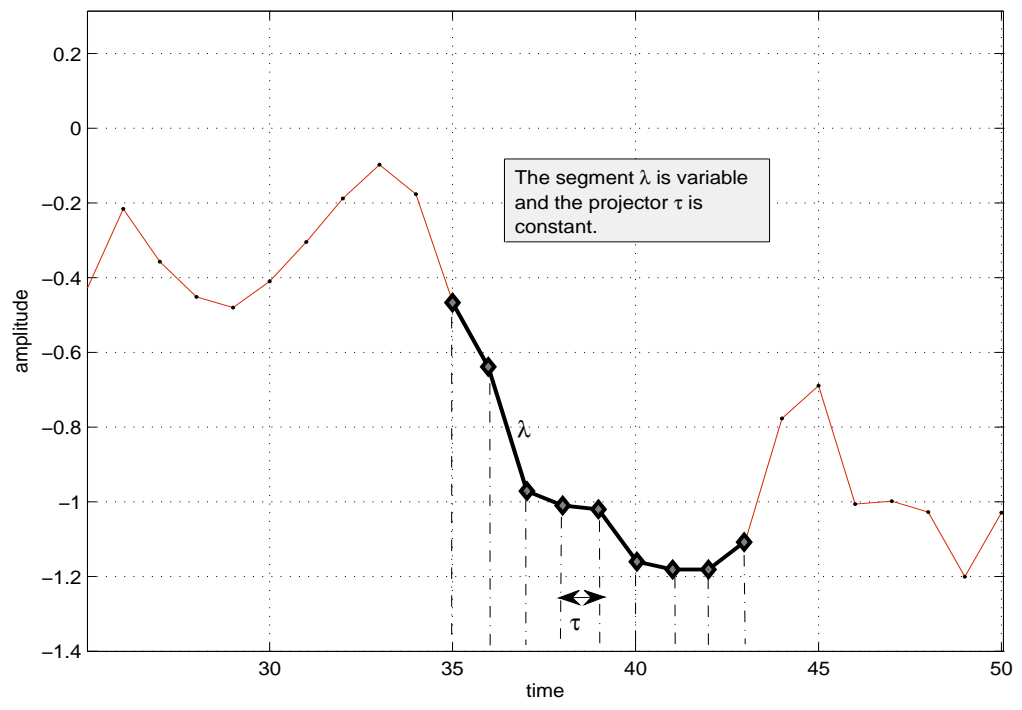

Figure 2: Principle of length measurement of a stochastic signal. 


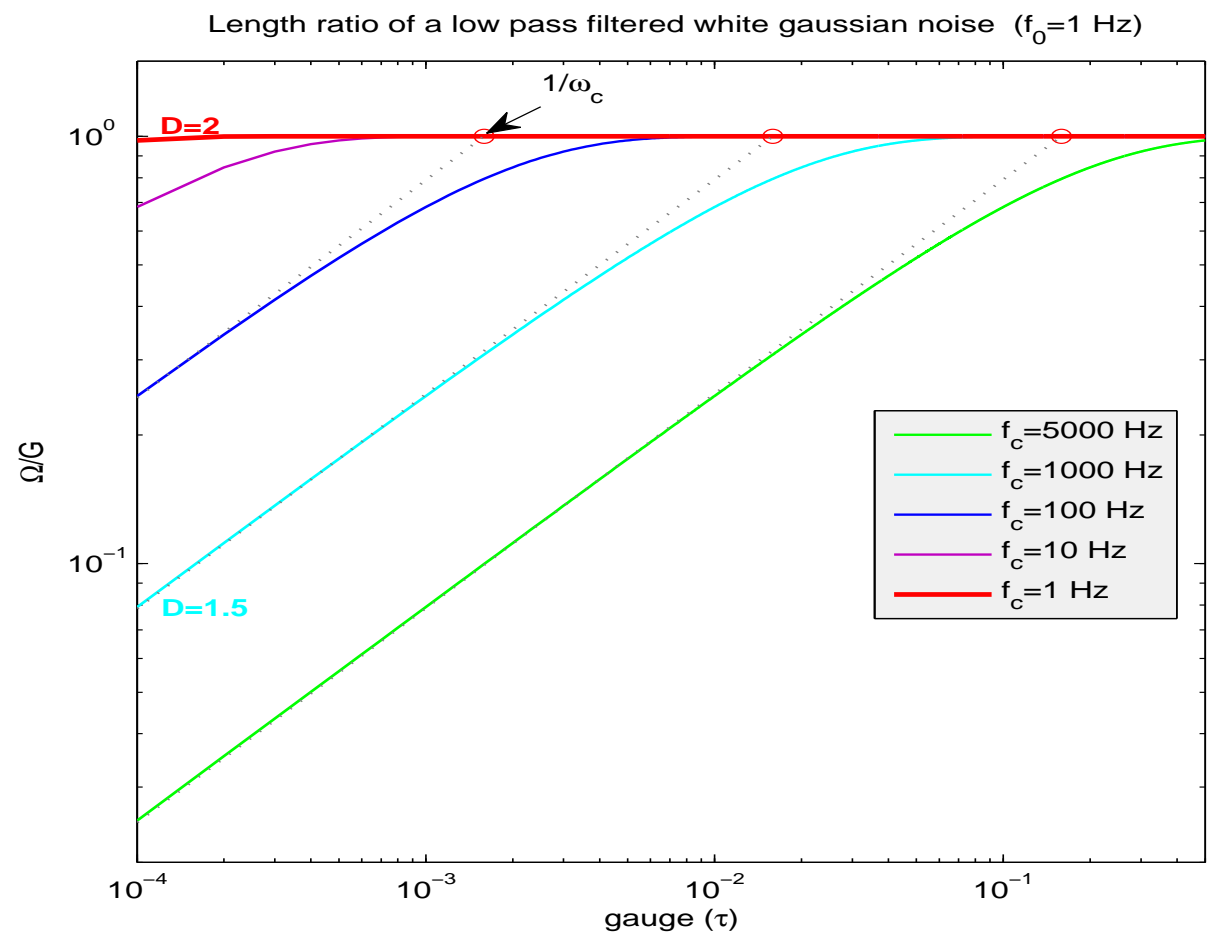

Figure 3: Length ratio of colored stochastic signals. First order Lorentzian low pass filter for different cut-off pulsations and for a fixed central pulsation. 


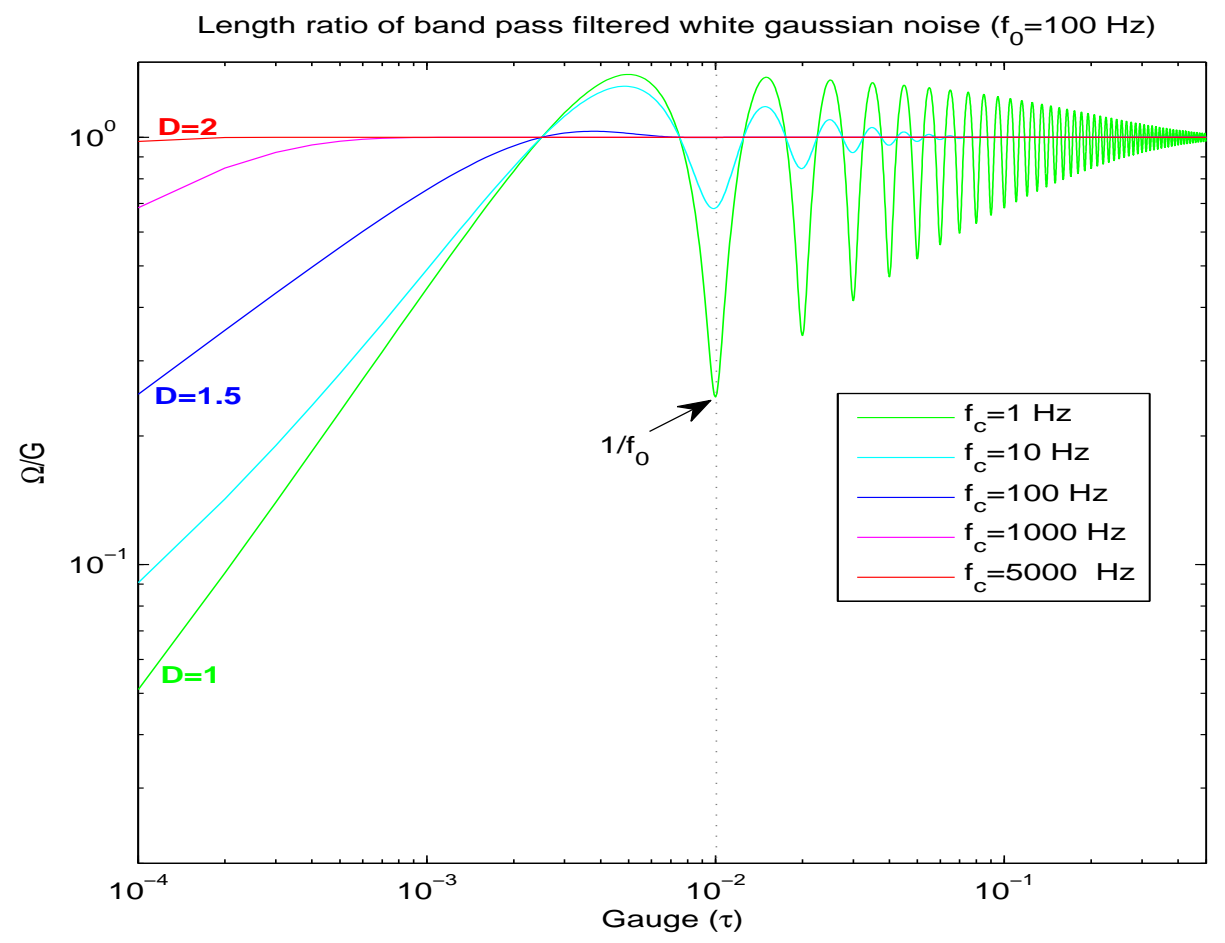

Figure 4: Length ratio of colored stochastic signals. First order Lorentzian low pass filter for different cut-off pulsations and for a fixed central pulsation. 

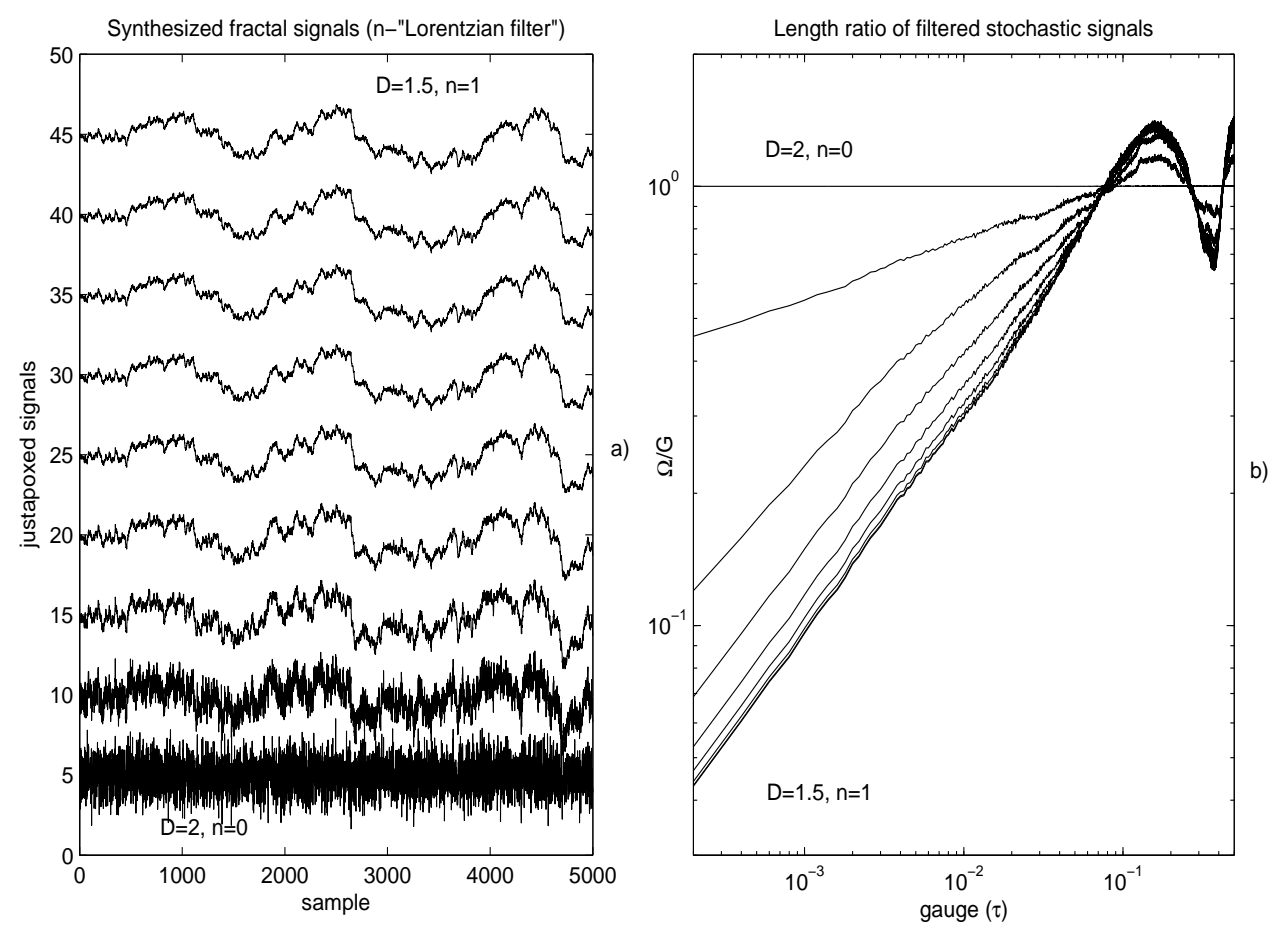

Figure 5: Length ratio of colored stochastic signals. a) Synthesized fractal signals with an $n$-order "Lorentzian filter". b) Loglog plot of Length ratio $\Omega / G$ versus gauge $\tau$. 